\title{
Rhoifolin Provides Neuroprotection against Spinal Cord Injury by Attenuation of Oxidative Stress, Inflammation and Apoptosis in Rats
}

Kangjian Long ${ }^{1}$, Xuehong Du ${ }^{1}$, Zhongxin $\mathrm{Yan}^{2}$, Jin $\mathrm{Xu}^{3}$

10.18805/IJAR.BF-1480

\begin{abstract}
Background: Rhoifolin is a plant flavonoid known to have antioxidant and anti-inflammatory properties. This study was taken to identify the effect of rhoifolin on Spinal cord injury (SCl) in a rat model.

Methods: Motor function of $\mathrm{SCl}$ rats was carried out by using in Basso, Beattie and Bresnahan test. Expression levels of different proteins such as IL-1 $\beta$, IL-6, TNF- $\alpha$, NF-кB p65, p-IкB- $\alpha$, p38MAPK, caspase-3 and caspase- 9 were carried out by using western blotting analysis. The Concentration of proteins was determined by using Bradford's method. Estimating of Glutathione (GSH), Glutathione peroxidase (GPx) and Superoxide dismutase (SOD) was carried out by using commercially available kit.

Result: Rhoifolin was tested at 20 and $40 \mathrm{mg} / \mathrm{kg}$ doses and showed a significant improvement in the motor function of $\mathrm{SCl}$ rats in Basso, Beattie and Bresnahan test. Moreover, rhoifolin treatment also attenuated pro-inflammatory cytokine levels such as IL-1 $\beta$, IL-6 and TNF- $\alpha$. The NF-KB pathway showed a significant attenuation as evident in the significant downregulation of the NF-kB p65 and $\mathrm{p}-\mathrm{\kappa} \mathrm{\kappa}-\alpha$ levels. In addition, rhoifolin treatment also caused a significant reduction in oxidative stress, as evident from changes in intracellular levels of Glutathione, GPx, MDA and SOD in the articular cartilage tissue. Further, we also tested rhoifolin for its effect on the apoptotic pathway by estimating the intracellular levels of p38MAPK, caspase- 3 and caspase- 9 . The current results show that rhoifolin has a neuroprotective in $\mathrm{SCl}$ rat model.
\end{abstract}

Key words: Cytokines, NF-kB pathway, Oxidative stress, Rhoifolin, Spinal cord injury.

\section{INTRODUCTION}

Spinal Cord Injury (SCI) is often caused by a physical injury that leads to infiltration of inflammatory cells and secondary degeneration (Ahn et al., 2015; Shin et al., 2013). SCI results in devastating impairment of neurological functions such as autonomic, motor and sensory dysfunction. Secondary injury in $\mathrm{SCl}$ results in lesions spreading out of the primary injury site leading to further damage (Aslan et al., 2009). The secondary injury in $\mathrm{SCl}$ is marked by increased inflammation and oxidative stress. Increased oxidative stress occurs when the level of reactive oxygen species (ROS) is more than the capacity of the antioxidant mechanism to scavenge them (Khayrullina et al., 2015; Paterniti et al., 2009). Moreover, the heightened production of inflammatory agents causes a strong inflammatory response (Genovese et al., 2009; Ni et al., 2014). It has been shown that attenuation of inflammatory signal helps in SCI recovery (Chen and Jin, 2016; Ji et al., 2014; Machova Urdzikova et al., 2016).

Mitogen-activated protein kinase (MAPK) is a factor in the signal transduction cascade for apoptosis (Pereira et al., 2013). The MAPK levels are generally a determiner of cell survival or death since their level reflects cell damage (Nafees et al., 2015; Yamaoka et al., 2012). Upregulated expression of MAPK has been reported in the glial cells and neurons in SCl (Ha et al., 2011; Lee et al., 2010). Moreover, MAPK is also involved in the inflammatory response (Bretón-Romero and Lamas, 2013).

The extant treatment regimens for $\mathrm{SCl}$ are largely ineffective and fail to result in complete recovery from

\begin{abstract}
1Department of Emergency, Ningbo Municipal Hospital of Traditional Chinese Medicine, Haishu district, Ningbo, Zhejiang Province, 315010, China.

2Department of gynaecology and Obstetrics, Shanghai General Hospital, Hongkou District, Shanghai, 200080, China.

${ }^{3}$ Department of Gastroenterology, Ningbo Municipal Hospital of Traditional Chinese Medicine, Haishu district, Ningbo, Zhejiang Province, 315010, China.
\end{abstract}

Corresponding Author: Jin Xu, Department of Gastroenterology, Ningbo Municipal Hospital of Traditional Chinese Medicine, Haishu district, Ningbo, Zhejiang Province, 315010, China.

Email: jinxu2040@gmail.com

How to cite this article: Long, K., Du, X., Yan, Z. and Xu, J. (2022). Rhoifolin Provides Neuroprotection against Spinal Cord Injury by Attenuation of Oxidative Stress, Inflammation and Apoptosis in Rats. Indian Journal of Animal Research. DOI: 10.18805/IJAR.BF-1480. Submitted: 20-12-2021 Accepted: 25-01-2022 Online: 03-03-2022

neurological and functional impairments (Varma et al., 2013). Flavonoids are bioactive compounds of plant origin with a 15 -carbon skeleton including two fused 6 -carbon rings (Merken and Beecher, 2000). Flavonoids in the human diet constitute a common group of polyphenolic compounds produced in plant cells as secondary metabolites. Flavonoids contain phenolic hydroxyl groups that mediate their antioxidant activity (Rice-Evans et al., 1996; Zheng et al., 2009), anti-inflammatory (Nijveldt et al., 2001) and the ability to scavenge free radicals (Cho et al., 2013). Flavonoids are 
known to show therapeutic potential in SCl (Zhang et al., 2017).

Rhoifolin is a flavanone first extracted from Rhus succedanea (Hattori and Matsuda, 1952). Rhoifolin has been shown to have anti-inflammatory, antioxidant (Eldahshan and Azab, 2012) and anticancer (Eldahshan, 2013) activity. Oxidative stress has an important role in progressing neurodegenerative disorders (Valko et al., 2007). Therefore, the antioxidative properties of rhoifolin may mediate its antioxidant and anti-inflammatory in SCl. Hence, the current study was planned to investigate the neuroprotective effect of rhoifolin on a rat model of $\mathrm{SCl}$.

\section{MATERIALS AND METHODS}

Experimental animals and estimation of rhoifolin toxicity

Male Sprague-Dawley rats (2 months old, weighing around $190-210 \mathrm{~g}$ ) were procured from the institutional animal housing facility. The experimental animals were kept under $12 / 12 \mathrm{~h}$ light/dark cycle under controlled conditions. The rats were fed with the standard rat diet and R.O. water as required. The animal experiments were approved by the Institutional Review Board of Shanghai University of Traditional Chinese Medicine and Institutional Animal Care and Use Committee of Shanghai University of Traditional Chinese Medicine (Approval No. JMU022/2019-22). This work was carried out in Shanghai University of Traditional Chinese Medicine from 2019-2021.

Rhoifolin (Sigma Aldrich USA) was dissolved in PBS and was administered in graded doses of 20,40,60, 80 and 100 $\mathrm{mg} / \mathrm{kg}$ via the intraperitoneal route to five rats each as mentioned by Zhao and his colleagues (Zhao et al., 2021). The animals were kept on a 10-day observation period. Health parameters such as diet, changes in weight, fluid intake and psyco-motor changes were measured. Rhoifolin did not show any toxicity at all the tested doses. Therefore, we selected the lowest two doses i.e. $20,40 \mathrm{mg} / \mathrm{kg}$ for further experiments.

\section{Establishment of rat SCI model and experimental groups}

For surgery, the rats were anesthetized by intraperitoneal administration of ketamine $(40 \mathrm{mg} / \mathrm{kg})$ and xylazine $(5.5 \mathrm{mg} / \mathrm{kg})$. Laminectomy of $T_{8}$ and $T_{9}$ was performed to remove vertebral peduncles and moderate contusion injury was executed using a modified Allen's weight drop apparatus on the exposed spinal cord with a weight of $10 \mathrm{~g}$ at a height of 55 $\mathrm{mm}$. Mechanical compression was confirmed by retraction flutter of legs, tail spasm and delayed paralysis.

The animals were randomly assigned to six experimental groups of 10 rats each: (1) Healthy group, no surgery (2) control group, with laminectomy + PBS (i.p.) without $\mathrm{SCl}$ (3) SCl group, SCl+PBS(i.p.) (4) MP group, treated with $55 \mathrm{mg} / \mathrm{kg}$ methyl prednisolone (i.p.) once a day for 5 days (5) $20 \mathrm{mg} / \mathrm{kg}$ rhoifolin, treated with $20 \mathrm{mg} / \mathrm{kg}$ rhoifolin (i.p.) once a day for 5 days (6) $40 \mathrm{mg} / \mathrm{kg}$ rhoifolin group, treated with $40 \mathrm{mg} / \mathrm{kg}$ rhoifolin (i.p.) once a day for 5 days. The experimental groups were established 12 hours after the establishment of SCI.

\section{Locomotor function test}

Locomotor activity was estimated by Basso, Beattie and Bresnahan (BBB) score method (Basso et al., 1995). BBB is a well-established method for the study of motor function after spinal cord contusion where a lower BBB score indicates a greater impairment of motor functions. The BBB scores range from 0 , signifying no hind limb movement to 21 signifying normal motor function. The BBB scores of the experimental groups were estimated at 24, 48, 72 hours and 7 days after spinal cord contusion. The technicians conducting BBB test were blinded to the group assignment using random codes.

\section{Estimation of water-content in the spinal cord}

The water-content in spinal cords was estimated after the commencement of drug treatment. The animals were euthanized with a $500 \mathrm{mg} / \mathrm{dose}$ of ketamine (IM) and spinal cords were excised and weighed. For dry weight, the spinal cord samples were dried for $24 \mathrm{~h}$ at $80^{\circ} \mathrm{C}$. The percentage of water-content in the spinal cords was calculated using the formula:

$$
\frac{\text { Wet weight }- \text { Dry weight }}{\text { Wet weight }} \times 100
$$

\section{Estimation of cytokine levels}

Blood was drawn by retro-orbital puncture of all experimental groups at the end of drug treatment scheduled. The blood was stored in anticoagulant and centrifuged at $3000 \mathrm{~g}$ for 30 min to collect the serum. The levels of TNF- $\alpha$, IL-1 $\beta$ and IL6 in the sera of CFA induced animals using ELISA kit (sigma bioscience, USA), following the manufacturer's instructions.

\section{Estimation of oxidative stress}

The animals were euthanized with a $500 \mathrm{mg} / \mathrm{dose}$ of ketamine (IM) and spinal cords were excised. $20 \mathrm{mg}$ of spinal cord tissue were taken and homogenized in PBS and centrifuged at $12500 \mathrm{~g}$ for $30 \mathrm{~min}$. The protein content of tissue supernatants was estimated using the Bradford's method and equal amount of protein was used for estimating the concentration of, Glutathione (GSH) using Glutathione GSH/GSSG Assay Kit (Sigma Aldrich, USA), Glutathione peroxidase (GPx) using Glutathione assay kit (Cayman Chemicals, USA), Malondialdehyde (MDA) by MDA assay kit (Abcam, USA) and Superoxide dismutase (SOD) by Superoxide Anion Assay Kit (Sigma Aldrich, USA). All the estimations were carried out following the respective manufacturer's protocols.

\section{Western blot analysis of MAPK}

Antibodies for NF-kB-p65, p-NF-kB-p65 and p38MAPK were procured from Santa Cruz Biotechnology, Inc. (USA). The anti-ІкB- $\alpha$ and anti-p-ІкB- $\alpha$ antibodies were purchased from the Cell Signaling Technology, Inc. (USA). An equal amount of total protein from spinal cord supernatants (described above) was electrophoresed in SDS-PAGE gel in three replicates and electrophoretically transferred on PVDF 
membranes. The membranes were washed with Trisbuffered saline which contained $0.2 \%$ Tween 20 (TBST) and blocked with $4 \%$ Non-fat dry-milk. Subsequently, primary antibodies were added with new blocking solution and incubated for 4 hours. HRP was added to the membranes after washing with Tris buffer saline. The membranes were developed with ECL detection system. The relative intensity of bands was analysed using ImageJ software (NIH, USA).

\section{Estimation of Caspase-3 and caspase-9 levels}

An equal amount of total protein from spinal cord supernatants was added with reaction buffer containing respectively AcLEHD-pNA and Ac-DEVD-pNA for caspase-9 and caspase3. The tubes were incubated for $2 \mathrm{~h}$ at $37^{\circ} \mathrm{C}$. Subsequently, the absorbance of reaction mixtures was recorded $405 \mathrm{~nm}$ on a spectrophotometer. The absorbance of treatment groups was compared with that of control groups.

\section{Statistical evaluation}

Individual mean \pm SEM were calculated from the data and the variance between the experimental groups was estimated using one-way ANOVA with Bonferroni's multiple corrections for post-hoc analysis. Significance values of
$\mathrm{P}<0.05$ were considered significant. All the statistical estimations were performed in Graph Pad Prism software.

\section{RESULTS AND DISCUSSION \\ Effect of rhoifolin on locomotor function and spinal cord water-content}

The locomotor function of experimental groups was determined using BBB scores (Fig 1). The BBB score was significantly reduced in the $\mathrm{SCl}$ groups comparing to the control in all the observation time points. A significant increase in BBB scores was observed in rhoifolin and MP groups in comparison to the $\mathrm{SCl}$ group. However, there was an insignificant difference in BBB scores between the rhoifolin groups as well as between the rhoifolin groups and the MP group.

The water content of spinal cord was estimated in the experimental groups (Fig 2). The SCI group had a significantly high water-content in comparison to the control group. Rhoifolin and MP groups and showed a significant decrease in spinal cord water content as compared to the $\mathrm{SCl}$ groups. However, there was o significant difference between the water content of rhoifolin groups and the MP group.

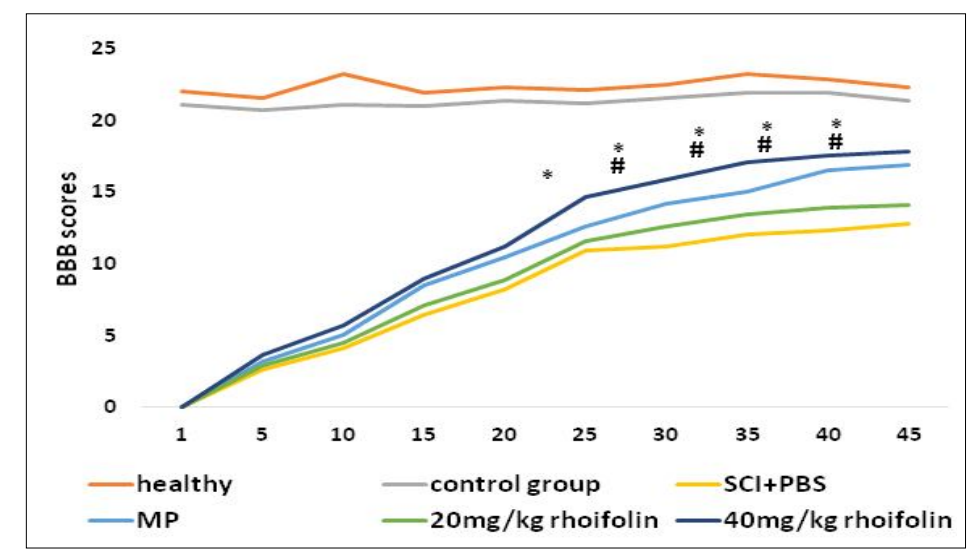

Fig 1: Effect of rhoifolin on BBB scores of $\mathrm{SCl}$ rats. ${ }^{*} \mathrm{P}<0.05$ represents the comparison between the $20 \mathrm{mg} / \mathrm{kg}$ rhoifolin-treated group and the vehicle-treated group; $\# \mathrm{P}<0.05$ represents the comparison between the $40 \mathrm{mg} / \mathrm{kg}$ rhoifolin-treated group and the vehicletreated group.

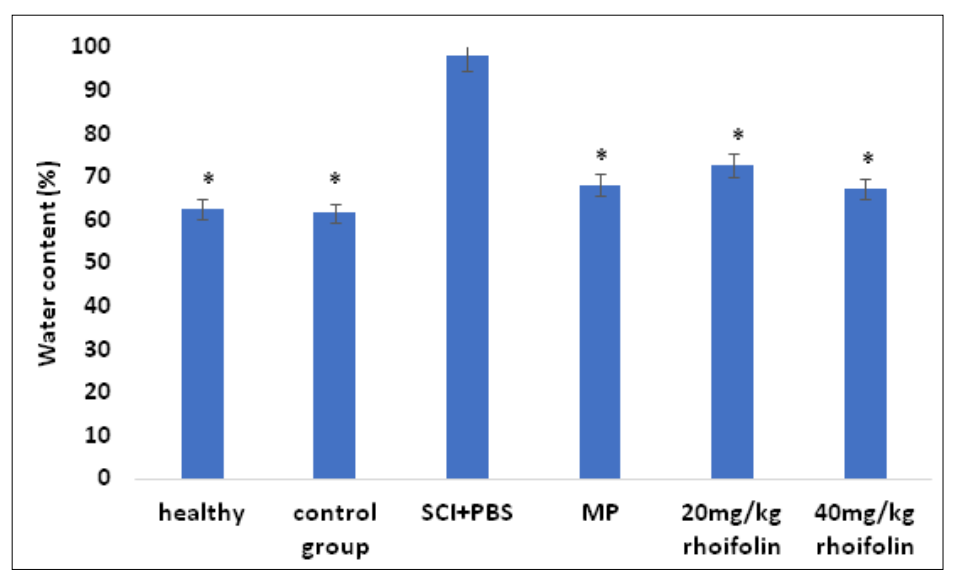

Fig 2: Effect of rhoifolin on spinal cord water content. * $\mathrm{P}<0.05$ vs. $\mathrm{SCl}$ group. 
Increased infiltration of proinflammatory cytokines and inflammatory response is a hallmark of the secondary injury in SCl. Rhoifolin treatment showed a significant attenuation of pro-inflammatory cytokine levels in $\mathrm{SCl}$ rats. The transcription factor NF-kB is a key mediator of inflammatory responses (Makarov, 2001). NF-kB also helps in the amplification of the inflammatory signal on the account of a cytokine feedback loop. Therefore, our results indicated that the anti-inflammatory and antioxidative properties of rhoifolin in $\mathrm{SCl}$ induced arthritis model are mediated by the NF-kB pathway. Previously rhoifolin has been reported to reduce inflammation in the rat edema model where it showed a significant attenuation of TNF- $\alpha$ levels (Eldahshan and Azab, 2012). Therefore, our findings anti-inflammatory effect of rhoifolin is consistent with previous studies.

\section{Effect of rhoifolin on pro-inflammatory cytokine and antioxidants levels}

ELISA analysis showed that the induction of $\mathrm{SCl}$ caused significantly increased blood sera TNF- $\alpha, \mathrm{IL}-1 \beta$ and IL-1 levels in comparison to the control (Fig 3). However, rhoifolin treatment caused a significant reduction of these cytokines as compared to the $\mathrm{SCl}$ group. It was also observed that cytokine levels in the highest rhoifolin concentration group were similar to the MP treatment group.
$\mathrm{SCI}$ induction significantly increased the oxidation levels in the spinal cord tissue (Fig 4). The levels of GSH, GPx, SOD was significantly downregulated and the levels of MDA was upregulated in $\mathrm{SCl}$ animals in comparison to the control. Rhoifolin treatment showed a significant improvement in the oxidation state in the articular tissue as compared to the $\mathrm{SCl}$ group animals. Moreover, the highest rhoifolin concentration group (40 mg/kg rhoifolin) showed an oxidation state at par with the MP group.

In addition to pro-inflammatory cytokines, oxidative stress is also a major factor in the progression of secondary injury in SCl (Khayrullina et al., 2015; Paterniti et al., 2009). The existence of high levels of ROS also indicates the degree of injury to the spinal cord and alleviation of oxidative stress has been suggested for therapeutic intervention in $\mathrm{SCl}$ (Jia et al., 2012). In this study, we used Glutathione, GPx, MDA and SOD levels as markers for the estimation of oxidative stress in the articular chondrocytes. Rhoifolin showed a strong antioxidant response in $\mathrm{SCl}$ animals, as evident from a significant decrease in these oxidative stress levels in the rhoifolin treatment groups. Previously, several other flavonoids have been reported to show antioxidative properties in SCI (Juurlink and Paterson, 1998). Therefore, we propose that rhoifolin can be as effective as other previously proposed flavonoids for SCI treatment.

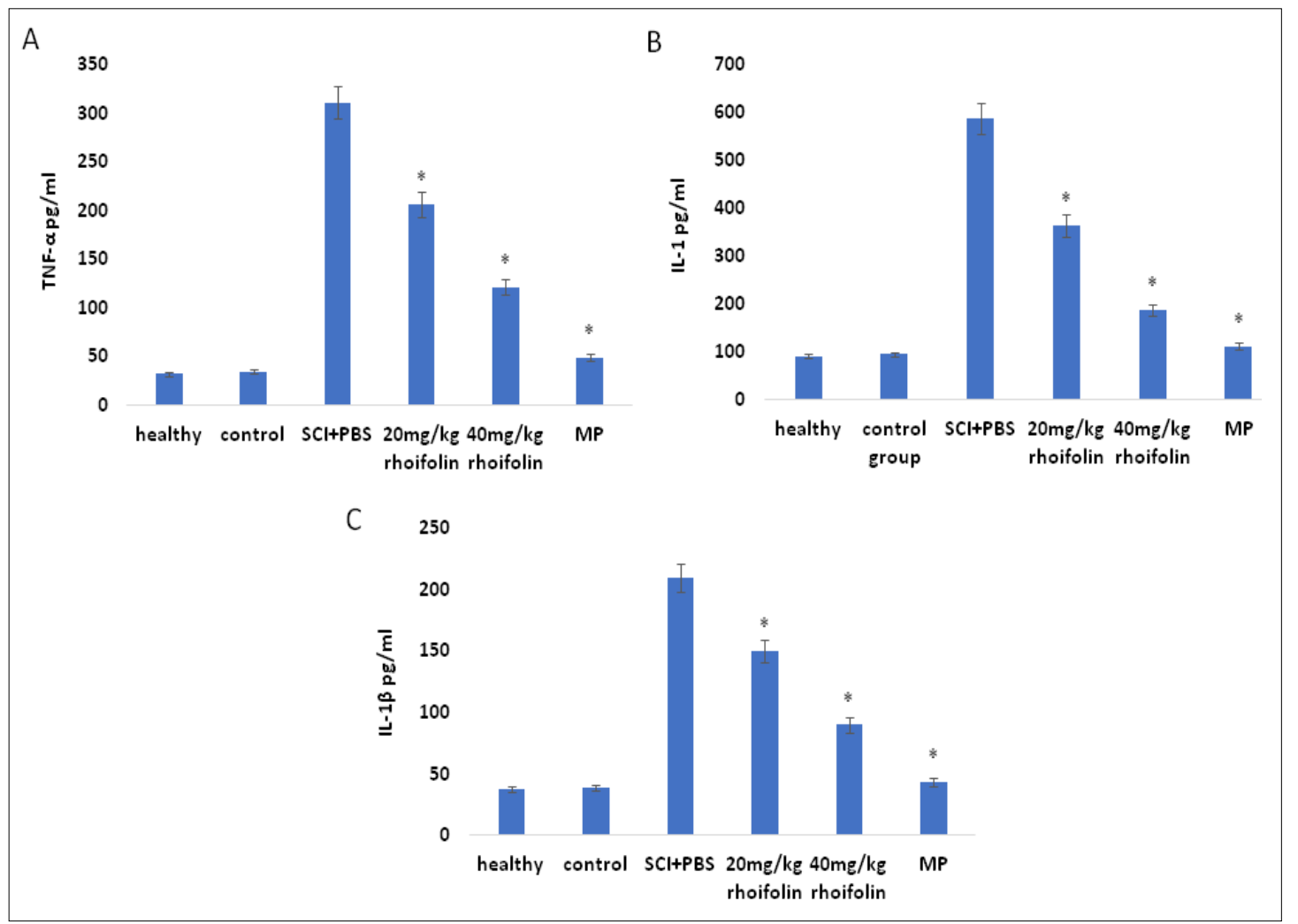

Fig 3: Effect of rhoifolin treatment levels of TNF- $\alpha(A) I L-1(B) I L-1 \beta(C)$. Data are expressed in mean $\pm S E M$. * $P \leq 0.05$ compared to SCI induced group. 


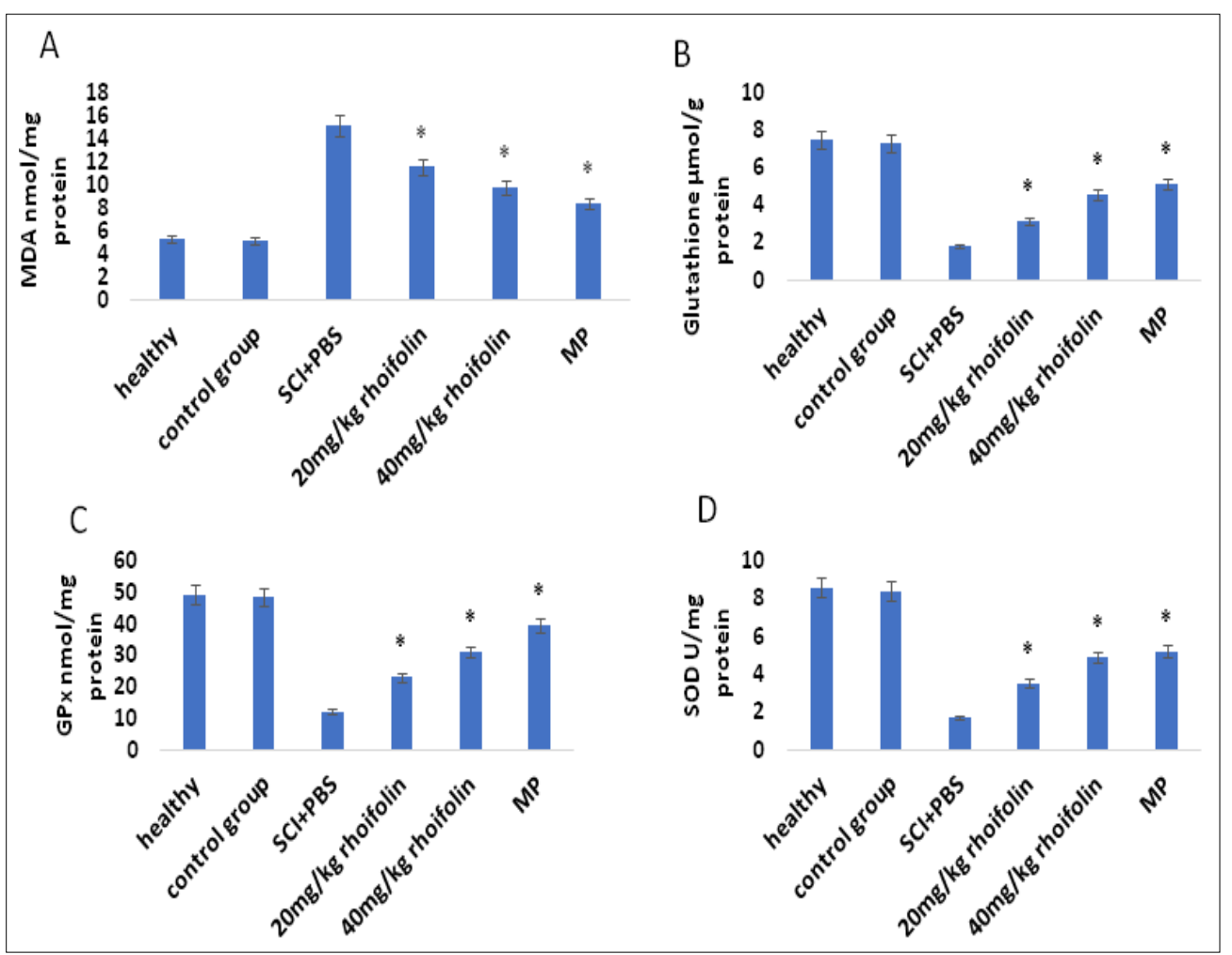

Fig 4: Effect of rhoifolin treatment levels of MDA (A) Glutathione (B) GPx (C) SOD (C) SOD (D). Data are expressed in mean \pm SEM. * $\mathrm{P} \leq 0.05$ compared to $\mathrm{SCl}$ group.

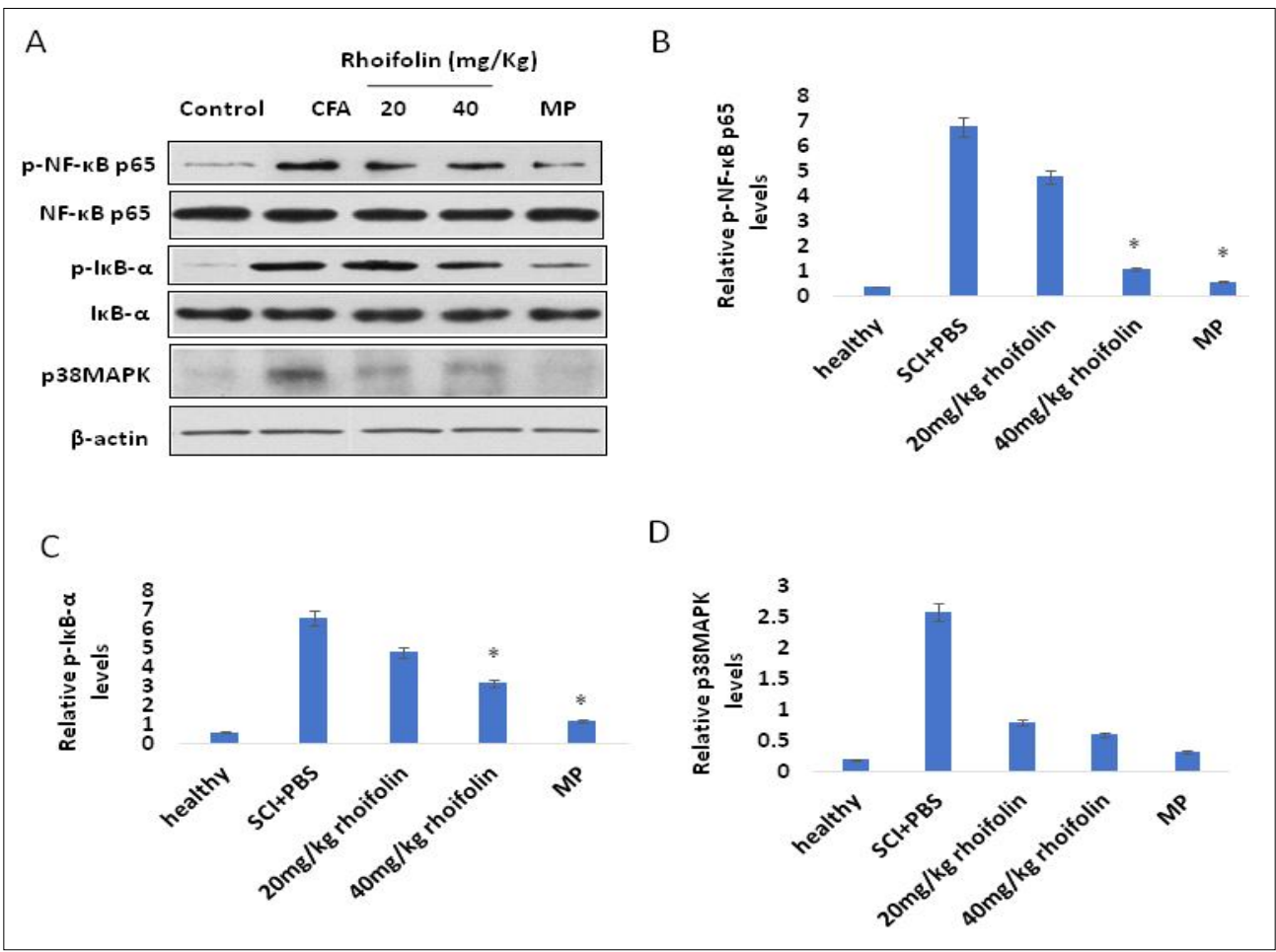

Fig 5: Western blot analysis of levels of phosphorylated and non-phosphorylated forms of NF-kB p65 and lêB-á and p38MAPK with $\beta$-actin used as a control (A). Western blot densitometry analysis of phosphorylated NF-êB p65 (B) Western blot densitometry analysis of phosphorylated lêB-á (C) Western blot densitometry analysis of p38MAPK (D). Data are expressed in mean \pm SEM. * $\mathrm{P} \leq 0.05$ compared to CFA induced group. 


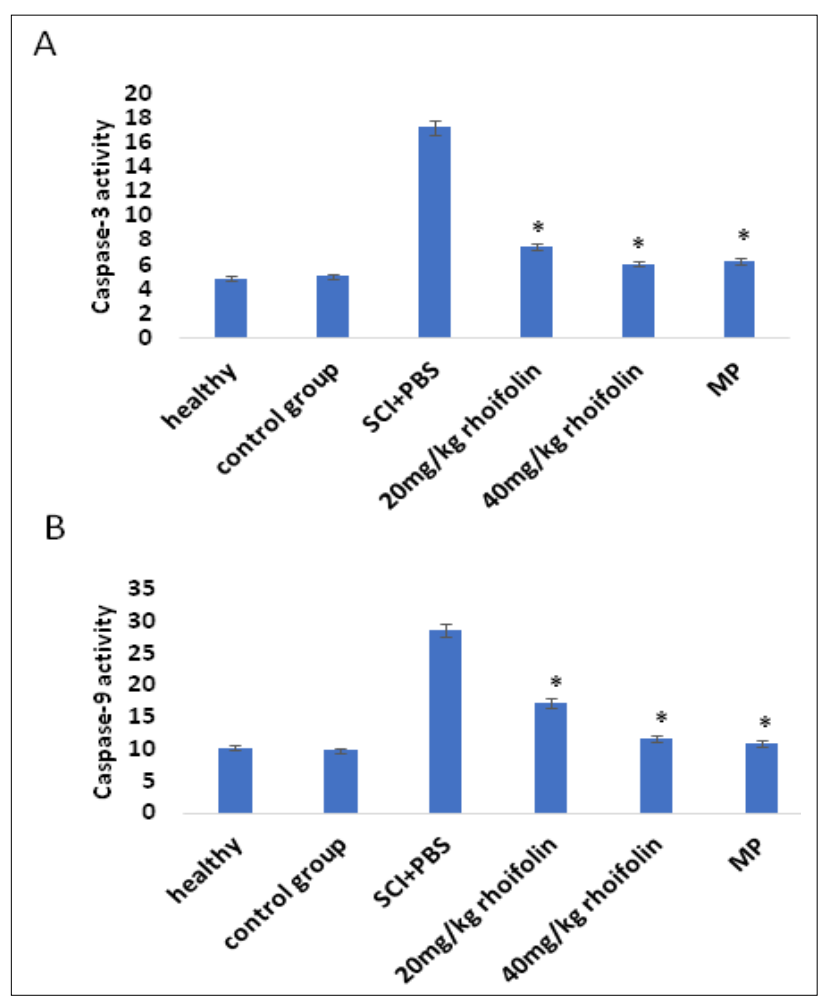

Fig 6: Effect of rhoifolin on Caspase-3 (A) and Caspase-9 (B)

levels. Data are expressed in mean \pm SEM. *P£0.05 compared to CFA induced group.

\section{Rhoifolin attenuates MAPK levels and downregulates caspase activity in $\mathrm{SCI}$ rats}

The effect of rhoifolin on the transcription factor NF-kB and p38MAPK was tested on the experimental groups. The western blot analysis showed a significantly large increase NF-кB-p65 and IкB- $\alpha$ and p38MAPK levels in spinal cord (Fig $5)$. Treatment with rhoifolin significantly decreased the levels of phosphorylated forms of NF-KB, IKB- $\alpha$ and p38MAPK.

Caspase- 9 and caspase- 3 activity was estimated in the experimental groups (Fig 6). These activities showed a significant upregulation in the $\mathrm{SCl}$ group in comparison to the control group. Rhoifolin treatment showed significant downregulation in the caspase activity. Moreover, the caspase activity in rhoifolin group was at par with that of MP group.

P38MPK is present in an inactive state in the cytoplasm of cells and is transported to the nucleus when activated (Tang et al., 2013). The phosphorylation cascade for activation of p38MAPK is triggered by external stimuli such as proinflammatory cytokines and free radical injury (Brunetti et al., 2013; Park et al., 2015; Zhu et al., 2013). The activated p38MAPK upregulates the expression of iNOS, COX and other inflammatory molecules resulting into the amplification of the inflammatory signal (Bretón-Romero and Lamas, 2013). Flavonoids have previously been suggested for arresting MAPK signaling in neurodegeneration (Schroeter et al., 2002). In this study, treatment with rhoifolin resulted in a significant downregulation of $\mathrm{p} 38 \mathrm{MAPK}$ indicating attenuation of the proapoptotic pathway. Moreover, this study also showed a significant reduction in caspase levels in $\mathrm{SCl}$ rats. $\mathrm{SCI}$ results in the induction of caspases which causes induction of apoptotic signaling pathway (Springer et al., 1999). Previously, the flavonoid apigenin has been reported to attenuate the levels of caspase-3 in SCl (Zhang et al., 2014). Therefore, we propose that rhoifolin is can be as effective as other flavonoids in the treatment of SCl.

\section{CONCLUSION}

In the present study, rhoifolin inhibited pro-inflammatory cytokines and the NF-KB pathways. Moreover, the intracellular oxidative stress was also significantly reduced upon rhoifolin treatment in SCI rats. This may have caused a reduction in apoptotic signal as observed in a reduction in the levels of p38MAPK and caspases. Overall, there was an improvement in the motor function in rhoifolin treated $\mathrm{SCl}$ rats. Therefore, the results of this study clearly show that rhoifolin can be a potentially safe alternative to extant therapies for $\mathrm{SCl}$ treatment. However, future studies will be mandated to identify the underlying mechanism of rhoifolin action on $\mathrm{SCl}$ such as the identification of its target molecules.

Conflict of interest: None.

\section{REFERENCES}

Ahn, M., Moon, C., Park, C., Kim, J., Sim, K.B. and Shin, T. (2015). Transient activation of an adaptor protein, disabled-2, in rat spinal cord injury. Acta Histochemica. 117: 56-61.

Aslan, A., Cemek, M., Buyukokuroglu, M.E., Altunbas, K., Bas, O., Yurumez, Y. and Cosar, M. (2009). Dantrolene can reduce secondary damage after spinal cord injury. European Spine Journal. 18: 1442-1451.

Basso, D.M., Beattie, M.S. and Bresnahan, J.C. (1995). A sensitive and reliable locomotor rating scale for open field testing in rats. J. Neurotrauma. 12: 1-21.

Bretón-Romero, R. and Lamas, S. (2013). Hydrogen peroxide signaling mediator in the activation of p38 MAPK in vascular endothelial cells. In Methods in Enzymology (Elsevier). pp. 49-59.

Brunetti, C., Di Ferdinando, M., Fini, A., Pollastri, S. and Tattini, M. (2013). Flavonoids as antioxidants and developmental regulators: Relative significance in plants and humans. International Journal of Molecular Sciences. 14: 3540-3555.

Chen, S. and Jin, Z. (2016). Local transplantation of human umbilical cord mesenchymal stem cells at different time after spinal cord injury in rats: A histological observation. Zhongguo Zuzhi Gongcheng Yanjiu. 20: 6714-6719.

Cho, J.G., Song, N.Y., Nam, T.G., Shrestha, S., Park, H.J., Lyu, H.N., Kim, D.O., Lee, G., Woo, Y.M., Jeong, T.S., et al. (2013). Flavonoids from the grains of C1/R-S transgenic rice, the transgenic Oryza sativa spp. japonica and their radical scavenging activities. J Agric Food Chem 61: 10354-10359.

Eldahshan, O.A. (2013). Rhoifolin; a potent antiproliferative effect on cancer cell lines. Br J Pharm Res 3: 46-53.

Eldahshan, O.A. and Azab, S.S. (2012). Anti-inflammatory effect of apigenin-7-neohesperidoside (rhoifolin) in carrageenininduced rat oedema model. Journal of Applied Pharmaceutical Science 2: 74. 
Genovese, T., Menegazzi, M., Mazzon, E., Crisafulli, C., Di Paola, R., Dal Bosco, M., Zou, Z., Suzuki, H. and Cuzzocrea, S. (2009). Glycyrrhizin reduces secondary inflammatory process after spinal cord compression injury in mice. Shock. 31: 367-375.

Ha, K.Y., Carragee, E., Cheng, I., Kwon, S.E. and Kim, Y.H. (2011). Pregabalin as a neuro protector after spinal cord injury in rats: Biochemical analysis and effect on glial cells. Journal of Korean Medical Science. 26: 404-411.

Hattori, S. and Matsuda, H. (1952). Rhoifolin, a new flavone glycoside, isolated from the leaves of Rhus succedanea. Archives of Biochemistry and Biophysics. 37: 85-89.

Huang, X., Wu, S., Wei, Y., Meng, S. and Jiang, R. (2021). Effect of the plant flavonoid, rhoifolin, on memory and cognition in a rat model of Alzheimer's disease Tropical Journal of Pharmaceutical Research. 20: 1481-1487.

Ji, W., Hu, S., Zhou, J., Wang, G., Wang, K. and Zhang, Y. (2014). Tissue engineering is a promising method for the repair of spinal cord injuries. Experimental and Therapeutic Medicine. 7: 523-528.

Jia, Z., Zhu, H., Li, J., Wang, X., Misra, H. and Li, Y. (2012). Oxidative stress in spinal cord injury and antioxidantbased intervention. Spinal Cord. 50: 264-274.

Juurlink, B.H. and Paterson, P.G. (1998). Review of oxidative stress in brain and spinal cord injury: suggestions for pharmacological and nutritional management strategies. The journal of Spinal Cord Medicine. 21: 309-334.

Khayrullina, G., Bermudez, S. and Byrnes, K.R. (2015). Inhibition of NOX2 reduces locomotor impairment, inflammation and oxidative stress after spinal cord injury. Journal of Neuroinflammation. 12: 172.

Lee, K.M., Jeon, S.M. and Cho, H.J. (2010). Interleukin-6 induces microglial CX3CR 1 expression in the spinal cord after peripheral nerve injury through the activation of p38 MAPK. European Journal of Pain. 14: 682. e681-682. e612.

Machova Urdzikova, L., Karova, K., Ruzicka, J., Kloudova, A., Shannon, C., Dubisova, J., Murali, R., Kubinova, S., Sykova, E. and Jhanwar-Uniyal, M. (2016). The antiinflammatory compound curcumin enhances locomotor and sensory recovery after spinal cord injury in rats by immunomodulation. International Journal of Molecular Sciences. 17: 49.

Makarov, S.S. (2001). NF-kappa B in rheumatoid arthritis: A pivotal regulator of inflammation, hyperplasia and tissue destruction. Arthritis Res. 3: 200-206.

Merken, H.M. and Beecher, G.R. (2000). Measurement of food flavonoids by high-performance liquid chromatography: A review. Journal of Agricultural and Food Chemistry. 48: 577-599.

Nafees, S., Rashid, S., Ali, N., Hasan, S.K. and Sultana, S. (2015). Rutin ameliorates cyclophosphamide induced oxidative stress and inflammation in Wistar rats: Role of NFKB/ MAPK pathway. Chemico-biological Interactions. 231: 98-107.

Ni, H., Jin, W., Yuan, B., Zhu, T., Wang, J., Jiang, J., Liang, W. and $\mathrm{Ma}, Z$. (2014). Curcumin inhibits the increase of labile zinc and the expression of inflammatory cytokines after traumatic spinal cord injury in rats. Journal of Surgical Research. 187: 646-652.

Nijveldt, R.J., van Nood, E., van Hoorn, D.E., Boelens, P.G., van Norren, K. and van Leeuwen, P.A. (2001). Flavonoids: A review of probable mechanisms of action and potential applications. Am J Clin Nutr. 74: 418-425.
Park, J., Min, J.S., Kim, B., Chae, U.B., Yun, J.W., Choi, M.S., Kong, I.K., Chang, K.T. and Lee, D.S. (2015). Mitochondrial ROS govern the LPS-induced pro-inflammatory response in microglia cells by regulating MAPK and NF-KB pathways. Neuroscience Letters. 584: 191-196.

Paterniti, I., Genovese, T., Crisafulli, C., Mazzon, E., Di Paola, R., Galuppo, M., Bramanti, P. and Cuzzocrea, S. (2009). Treatment with green tea extract attenuates secondary inflammatory response in an experimental model of spinal cord trauma. Naunyn-Schmiedeberg's Archives of Pharmacology. 380: 179-192.

Pereira, L., Igea, A., Canovas, B., Dolado, I. and Nebreda, A.R. (2013). Inhibition of p38 MAPK sensitizes tumour cells to cisplatin induced apoptosis mediated by reactive oxygen species and JNK. EMBO Molecular Medicine. 5: 1759-1774.

Rice-Evans, C.A., Miller, N.J. and Paganga, G. (1996). Structureantioxidant activity relationships of flavonoids and phenolic acids. Free Radic Biol Med. 20: 933-956.

Schroeter, H., Boyd, C., Spencer, J.P., Williams, R.J., Cadenas, E. and Rice-Evans, C. (2002). MAPK signaling in neurodegeneration: Influences of flavonoids and of nitric oxide. Neurobiology of Aging. 23: 861-880.

Shin, T., Ahn, M., Moon, C., Kim, S. and Sim, K.B. (2013). Alternatively activated macrophages in spinal cord injury and remission: another mechanism for repair? Molecular Neurobiology. 47: 1011-1019.

Springer, J.E., Azbill, R.D. and Knapp, P.E. (1999). Activation of the caspase- 3 apoptotic cascade in traumatic spinal cord injury. Nature Medicine. 5: 943.

Tang, N., Zhang, Y.P., Ying, W. and Yao, X.X. (2013). Interleukin$1 \beta$ upregulates matrix metalloproteinase- 13 gene expression via c-Jun $\mathrm{N}$-terminal kinase and p38 MAPK pathways in rat hepatic stellate cells. Molecular Medicine Reports. 8: 1861-1865.

Valko, M., Leibfritz, D., Moncol, J., Cronin, M.T., Mazur, M. and Telser, J. (2007). Free radicals and antioxidants in normal physiological functions and human disease. Int $\mathrm{J}$. Biochem Cell Biol 39: 44-84.

Varma, A.K., Das, A., Wallace, G., Barry, J., Vertegel, A.A., Ray, S.K. and Banik, N.L. (2013). Spinal cord injury: A review of current therapy, future treatments and basic science frontiers. Neurochemical Research. 38: 895-905.

Yamaoka, G., Morino, T., Morizane, K., Horiuchi, H., Miura, H. and Ogata, T. (2012). p38 mitogen-activated protein kinase inhibitor reduces neurocan production in cultured spinal cord astrocytes. Neuro Report. 23: 546-550.

Zhang, F., Li, F. and Chen, G. (2014). Neuroprotective effect of apigenin in rats after contusive spinal cord injury. Neurological Sciences. 35: 583-588.

Zhang, P., Hölscher, C. and Ma, X. (2017). Therapeutic potential of flavonoids in spinal cord injury. Reviews in the Neuro Sciences. 28: 87-101.

Zheng, L., Kagedal, K., Dehvari, N., Benedikz, E., Cowburn, R., Marcusson, J. and Terman, A. (2009). Oxidative stress induces macroautophagy of amyloid beta-protein and ensuing apoptosis. Free Radic Biol Med. 46: 422-429.

Zhu, J., Luo, C., Wang, P., He, Q., Zhou, J. and Peng, H. (2013). Saikosaponin A mediates the inflammatory response by inhibiting the MAPK and NF-KB pathways in LPSstimulated RAW 264.7 cells. Experimental and Therapeutic Medicine. 5: 1345-1350. 